\title{
Correction to: Pluripotent Stem Cell-Derived Human Tissue: Platforms to Evaluate Drug Metabolism and Safety
}

\author{
Jose Meseguer-Ripolles, ${ }^{1}$ Salman R. Khetani, ${ }^{2}$ Javier G. Blanco, ${ }^{3}$ Miari Iredale, ${ }^{1}$ and David C. Hay ${ }^{1,4}$
}

Published online 12 February 2018

Correction to: AAPS J

https://doi.org/10.1208/s12248-017-0171-8

The original version of the published article contains errors throughout the text, which were introduced by the typesetter when performing the author's proof corrections. The original article was corrected.

The online version of the original article can be found at https:// doi.org/10.1208/s12248-017-0171-8

${ }^{1}$ MRC Centre for Regenerative Medicine, 5 Little France Drive, Edinburgh, EH16 4UU, UK.

${ }^{2}$ University of Illinois at Chicago, Bioengineering (MC 063), $851 \mathrm{~S}$ Morgan St, 218 SEO, Chicago, Illinois 60607, USA.

${ }^{3}$ School of Pharmacy and Pharmaceutical Sciences, University at Buffalo, The State University of New York, Buffalo, New York, USA.

${ }^{4}$ To whom correspondence should be addressed. (e-mail: davehay@talktalk.net) 